\title{
Aplicação da Teoria de Expansão Assintótica Uniforme à Acústica em Águas Profundas
} Rosana B. Santiago - IF/UERJ, Luiz G. Guimarães - IF/UFRJ.

\section{Copyright 2006, SBGf - Sociedade Brasileira de Geofísica}

Este texto foi preparado para a apresentação no II Simpósio de Geofísica da Sociedade Brasileira de Geofísica, Natal, 21-23 de setembro de 2006. Seu conteúdo foi revisado pela Comissão Tecno-científica do II SR-SBGf mas não necessariamente representa a opinião da SGBf ou de seus associados. É proibida a reprodução total ou parcial deste material para propósitos comerciais sem prévia autorização da SBGf.

\section{Resumo}

Vários modelos de acústica oceanográfica são baseados em perfis multicamadas caracterizados pela densidade do fluído ou meio elástico bem como pela velocidade do som neste meio. Dentre esses modelos, o modelo de guia de onda sonora de Pekeris é um dos mais simples, pois descreve a propagação do som entre a superfície e o fundo do oceano com um modelo de apenas duas camadas. Em geral, o modelo de Pekeris fornece resultados satisfatórios quando modela "águas rasas" ou quando não há grande variação da velocidade do som com a profundidade. Quando isto não ocorre, tenta-se estratificar a região entre a superfície e o fundo do oceano em um número maior de camadas, bem como também adotando-se uma dependência linear da velocidade do som com a profundidade. Entretanto, tal procedimento aumenta o grau de dificuldade tanto teórico quanto numérico de resolução do problema, podendo as vezes inclusive tais soluções apresentarem resultados espúrios. Neste trabalho mostraremos que podemos tentar contornar um pouco essas dificuldades, aplicando a teoria de expansões assintóticas para as soluções da equação de Helmholtz tendo agora a velocidade sonora uma dependência funcional explícita com a profundidade (Modelo de Pekeris generalizado). Mostraremos também que essa teoria é estável e acurada. Palavras-Chave: acústica oceanográfica, guias de onda, águas profundas, expansões assintóticas.

\section{Introdução:}

A determinação do comportamento da velocidade do som no oceano é uma tarefa extremamente complexa, pois tal velocidade depende de vários fatores tais como pressão, temperatura e salinidade [1]. Estes fatores variam não só geograficamente como sazonalmente tornando tal estudo uma tentativa de modelagem "quase local" dos mesmos. Por outro lado, como em média a maior variação destes fatores ocorre com a variação da profundidade oceânica, tem-se que a magnitude $c$ da velocidade da som é também uma função da profundidade. Além disso o som é uma onda e dependendo do perfil de como $c$ varia com a profundidade, alguns efeitos ondulatórios relacionados ao transporte de energia e momentum podem se manifestar. Por exemplo, pode haver o aparecimento de regiões de grandes gradientes de pressão (cáusticas sonoras), difração por obstáculos bem como o surgimento de regiões e direções de "fácil" propagação, nas quais a energia é preferencialmente transportada. Estes últimos são chamados de guias de onda e serão o principal objeto de estudo deste trabalho. Para tanto o artigo será organizado da seguinte forma, na seção a seguir apresentaremos o modelo de Pekeris e sua generalização para águas profundas. Além disso, baseando-se na teoria de expansões assintóticas uniformes (EAU), mostraremos como é possível se resolver de uma maneira acurada este tipo de problema. Na seção restante comentaremos nossos resultados e possíveis perspectivas futuras.

\section{Teoria de EAU para o Modelo de Pekeris Generalizado:}

É bem sabido que para fluídos com densidade constante ou constante por partes, os campos de pressão e de deslocamento que evoluem harmonicamente com o tempo $(\exp (i \omega t))$ satisfazem nestes meios à equação de Helmholtz [2, 3, 4]:

$$
\left(\nabla^{2}+\frac{\omega^{2}}{c(\mathbf{r})^{2}}\right) \Phi(\mathbf{r})=0
$$

Vamos admitir aqui um sistema de coordenadas cilíndrico $(r, \phi, z)$, onde superfície e profundidade do oceano são respectivamente caracterizadas pelo plano $z=0$ e direção positiva de $z$. Sendo assim, se $c$ depende apenas da profundidade $z$ e propondo que o campo $\Phi$ possua simetria azimutal, então as soluções finitas em $r=0$ da eq(1) possuem a forma $\Phi(\mathbf{r}) \equiv J_{0}(k r) \Psi(z)$. Onde $k$ é a constante de separação, $J_{0}$ é a função cilíndrica de Bessel de ordem zero e $\Psi$ satisfaz a seguinte equação de onda:

$$
\frac{d^{2} \Psi}{d z^{2}}+\left(\frac{\omega^{2}}{c(z)^{2}}-k^{2}\right) \Psi=0 .
$$

Em sua forma mais simples o modelo de Pekeris[5] consiste em supor o oceano como dois fluídos embebidos em um semi-espaço infinito, onde a superfície do oceano é uma fronteira acusticamente "rígida", pois despreza-se a propagação sonora no ar em detrimento a no fluído. Neste modelo, logo após a superfície e até uma profundidade $D$, se supõe um fluído de densidade $\rho_{1}$ no qual o som se propaga com velocidade $c_{1}$. Na camada semiinfinita seguinte, admite-se um outro fluído com densidade $\rho_{2}$ e velocidade do som $c_{2}$. As propriedades ondulatória e elástico-acústicas dos meios são conservadas exigindo-se que os campos de pressão e deslocamento sejam contínuos nas interfaces entre os fluídos em $z=D$. No caso em que $c_{1}<c_{2}$ e $\rho_{1}<\rho_{2}$, mostra-se que 
$[2,3,5]$ para alguns valores discretos de $k$ a energia se propaga eficientemente entre a superfície até basicamente à profundidade $D$, e que a partir dai tem-se uma quase "penumbra" acústica. Neste sentido, diz-se que esse sistema se comporta como uma guia de ondas. Esses guias em"águas rasas" são muito sensíveis à variações externas principalmente as sazonais. De modo que a procura e o estudo de guias mais estáveis em "águas profundas" se tornou fundamental. Infelizmente, o sucesso do modelo de Pekeris restringe-se à "águas rasas", onde $D$ não ultrapassa algumas centenas de metros [2]. Inviabilizando assim a utilização deste modelo para discrição de guias de longo alcance em"águas profundas". Uma tentativa de superar isto consiste em introduzir um número maior de camadas à medida que a profundidade aumenta. Entretanto se o número de camadas aumenta muito, tal procedimento aumenta também a complexidade computacional do problema [2]. Além disso, dependendo da razão entre o comprimento de onda incidente e largura das camadas, tal método pode introduzir resultados expúrios ao problema real [2]. Em vista disso, uma outra alternativa consiste em tratar as propriedades acústicas desses meios inomogêneos modelando-se uma dependência funcional da velocidade do som com a profundidade. A este último procedimento chamaremos de modelo de Pekeris generalizado que passaremos agora a descrever para o caso de possíveis guias em "águas profundas". Para tanto, vamos admitir que $c(z)=c_{0} f(z)$, onde $c_{0}$ é a velocidade do som emediatamente após a superfície oceânica e $f$ é uma função bem comportada da profundidade. Possíveis modelos de funções interpoladoras $f$ são bastante estudados e conhecidos na literatura $[6,7,8]$. Em geral, no caso de guias essas funções devem apresentar um valor de velocidade mínimo $c_{\min }$ para uma dada profundidade finita $z_{m i n}$ [2], como mostra a figura(1). Aqui vamos modelar o perfil de velocidade mostrado na figura(1) da seguinte maneira:

$$
c(z)=\frac{c_{0}}{\sqrt{1+A / z_{0}}} \sqrt{1+\frac{A}{z+z_{0}}+\frac{\tanh (B z / L)}{B}} .
$$

Onde os parâmetros $z 0, A, B$ e $L$ são ajustados a um dado pefil de oceano específico. No caso limite de $L \rightarrow \infty$, a eq(3) se reduz ao bem conhecido modelo de meio acústico linear[2, 3]. Entretanto, em geral não é possível se obter uma solução explícita da eq(2) para o perfil de velocidade(3). Pode-se tentar resolver numericamente a eq(2), mas como veremos mais adiante, isto não é uma tarefa fácil e nem sempre seus resultados são satisfatórios. Neste trabalho vamos tentar contornar um pouco isso resolvendo o problema acima utilizando a técnica desenvolvida por F. W. J. Olver[9], baseada no desenvolvimento de EAU para solução de equações diferenciais ordinárias. Esta técnica é um aprimoramento do método WKB[9], principalmente por suas soluções serem finitas nos pontos $z_{1}$ e $z_{2}$ que são zeros da equação,

$$
c\left(z_{1,2}\right) k=\omega
$$

De tal forma que, por exemplo, uma possível solução de
(2) na região $z_{1} \leq z \leq z_{\min }$ é descrita na teoria EAU por:

$$
\Psi(z) \approx \Psi_{0} A i\left(z_{1}+\left[\frac{3}{2 \alpha_{1}} \int_{z 1}^{z} d z^{\prime} \sqrt{\left|\frac{\omega^{2}}{c\left(z^{2}\right)^{2}}-k_{n}^{2}\right|}\right]^{2 / 3}\right)
$$

Onde $A i$ são as funções de Airy[10], $\Psi_{0}$ e $\alpha_{1}$ são constantes, sendo que esta última depende fortemente do valor da velocidade nos pontos $z_{1}$ e $z_{2}$. Além disso, a constante de separação $k \rightarrow k_{n}$ admite agora apenas valores discretos que satisfazem assintoticamente a:

$$
\int_{z 1}^{z_{2}} d z^{\prime} \sqrt{\left|\frac{\omega^{2}}{c(z)^{2}}-k_{n}^{2}\right|} \approx\left(n+\frac{1}{2}\right) \pi ; n=0,1,2, \ldots
$$

De modo que, para uma dada freqüência fixa $\omega$, resolvendo-se simultaneamente as eqs(4 e 6) para $k \rightarrow$ $k_{n}$, a fórmula(5) representa a EAU da solução de (2) no intervalo $z_{1} \leq z \leq z_{\min }$ [9]. Vejamos agora, um exemplo numérico disto.

\section{Resultados:}

Vamos supor que para um dado modelo de oceano, para uma profundidade $z$ medida em metros, a velocidade do som (em metros por segundo) seja interpolada por:

$$
\begin{aligned}
c(z) \approx & 1295.642269\left[1+\frac{269.3576456}{z+652.562325}+\right. \\
& \left.\frac{\tanh (3 z / 7833.218557)}{3}\right]^{1 / 2}(\mathrm{~m} / \mathrm{s}) .
\end{aligned}
$$

Com $c_{0} \approx 1540 \mathrm{~m} / \mathrm{s}, c_{\min } \approx 1468 \mathrm{~m} / \mathrm{s}$ e $z_{\min } \approx$ $884 \mathrm{~m}$. Para uma freqüência $\omega=50 \mathrm{rad} / \mathrm{s}$, a figura(2a e b) mostra para os estados $n=0$ e 1 respectivamente, o comportamento do campo acústico $\Psi$ (em unidades arbitrárias) a medida que a profundidade $z$ varia. Nesta figura(2a e b), a curva cheia é a teoria EAU associada ao perfil de velocidade(7) enquanto que os círculos representam os resultados obtidos por integração numérica (IN) de (2) utilizando-se o método de Runge-Kutta de $4^{a}$ ordem. Nota-se que, com execeção dos pontos na vizinhança de $z_{\text {min }} \approx 884 \mathrm{~m}$, o método IN é instável e rapidamente apresenta resultados não confiáveis uma vez que sabemos que para $\mathrm{n}=0, \Psi$ deve apresentar um único pico na vizinhança de $z_{\text {min }}$, enquanto que para $\mathrm{n}=1, z_{\text {min }}$ é próximo de um zero de $\Psi$ e que $\Psi$ deve apresentar apenas um valor máximo e outro mínimo [2, 3]. Por outro lado, observase da figura(2a e b) que a teoria EAU é bastante estável para qualquer valor de profundidade $z$ e apresenta resultados bem satisfatórios, uma vez que todos esses critérios para o comportamento de $\Psi$ são satisfeitos.

\section{Conclusão e Perspectivas Futuras:}

Mostramos aqui que o método de EAU pode se tornar uma ferramenta útil em acústica oceanográfica, principalmente no estudo de guias em "águas profundas". Onde tal método aparece como uma alternativa estável para 
o cálculo de campos elástico-acústicos em meios inomogêneos isotrópicos. Uma das grandes vantagens deste método consiste em sua convergência uniforme[9]. Em outras palavras, a garantia de convergência uniforme é uma ferramenta poderosa não apenas teórica como experimentalmente, pois uma vez que a fórmula de extraploção(3) possui poucos parâmetros de ajuste (4 ao todo, $z 0, A, B$ e $L$ ), pode-se partir de alguns resultados de medida de velocidade em "águas rasas" tentar estimar o comportamento da velocidade $c$ em "águas profundas". Posteriormente, a eficiência e acurácia deste procedimento pode ser confrontada com resultados experimentais para o perfil do campo elástico-acústico, modelandose este através da eq(5). Por outro lado, baseados em nossos trabalhos anteriores em eletromagnetismo e métodos matemáticos $[11,12,13,14,15,16,17,18]$, acreditamos que é possível se estender esta teoria não apenas ao problema do guia, que envolve um espectro discreto de $k_{n}$, como também a problemas relacionados ao espectro contínuo, tipo propagação, difração e espalhamento por obstáculos no âmbito da acústica oceanográfica. Estes são nossos esforços atuais, e que pretendemos relatar em breve.

\section{Agradecimentos}

Os autores agradecem ao CNPq e FAPESB pelo apoio financeiro parcial e um dos autores (LGG) agradece aos Profs Roberto Argollo e Olivar Lima pelos esforços dispensados e agradável acolhida no CPGG-UFBA.

\section{Referências}

[1] W. D. Wilson, J. Acoust. Soc. Am. 23, 1357 (1960).

[2] F. B. Jensen, W. A. Kuperman, M. B. Porter, H. Schmidt, Computational Ocean Acoustics, (AIP Press, New York, 2000).

[3] I. Tolstoy, C. S. Clay, Ocean Acoustics: Theory and Experiments in Underwater Sound, (Mc G. Hill, New York, 1966).

[4] C. S. Clay, H. Medwin, Acoustical Oceanography: Principles and Applications, (Willey, New York, 1977).

[5] C. L. Pekeris, Geol. Soc. Am. Mem. 27, (1948).

[6] D. S. Jones, Phil. Trans. R. Soc. Lond. 255, 341 (1963).

[7] M. A. Perdersen, D. F. Gordon, J. Acoust. Soc. Am. 51, 323 (1972).

[8] F. R. Dinapoli, R. L. Deavenport, J. Acoust. Soc. Am. 67, 92 (1980).

[9] F. W. J. Olver, Asymptotics and Special Functions, (A. Press, N. York 1974)

[10] M. Abramowitz, I. Stegun, eds., Handbook of Mathematical Functions (Dover, New York, 1968).

[11] L. G. Guimarães, H. M. Nussenzveig, "Theory of Mie Resonances and Ripple Fluctuations", Optics Communications 89, 363 (1992).

[12] L. G. Guimarães, "Theory of Mie Caustics", Optics Communications 103, 339 (1993).

[13] L. G. Guimarães, H. M. Nussenzveig, "Uniform Approximations to Mie Resonances", Journal of Modern Optics 41, 625 (1994).
COMPORTAMENTO DA VELOCIDADE DO SOM COM A PROFUNDIDADE

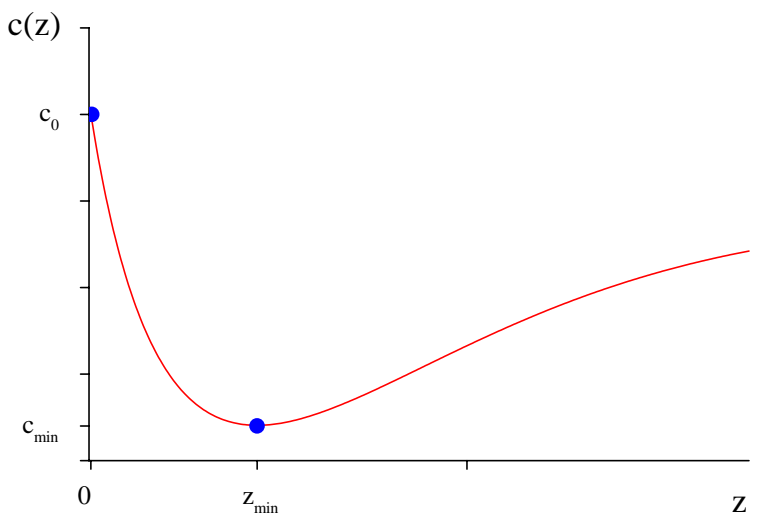

Figura 1: mostra que $c(z)$ apresenta um valor mínimo $c_{\text {min }}$ em $z=z_{\min }$.

[14] A.G. Simão, L.G. Guimarães, G. Videen, "A comparative study in resonant light scattering between spherical and cylindrical dielectric hosts with a metallic inclusion", J. of Quant. Spectroscopy and Radiative Transfer 70, 761 (2001).

[15] P.C.G. de Moraes, L. G. Guimarães, "Semiclassical theory to optical resonant modes of a transparent dielectric spheroidal cavity", Applied Optics 41, 2955 (2002).

[16] A. G. Simão, L. G. Guimarães, J. P. R. F. Mendonça, "Evanescent wave coupling in light scattering of an off-axis normally incident Gaussian beam by two parallel nonabsorbing cylinders", J. Opt. Soc Am. A 19, 2053 (2002).

[17] P.C.G. de Moraes, L. G. Guimarães, "Uniform asymptotic formulae for the spheroidal angular function", J. of Quant. Spectroscopy and Radiative Transfer 74, 757 (2002).

[18] P.C.G. de Moraes, L. G. Guimarães, "Uniform asymptotic formulae for the spheroidal radial function", J. of Quant. Spectroscopy and Radiative Transfer 79, 973 (2003). 
COMPARAÇÃO ENTRE AS TEORIAS EAU E IN

a)

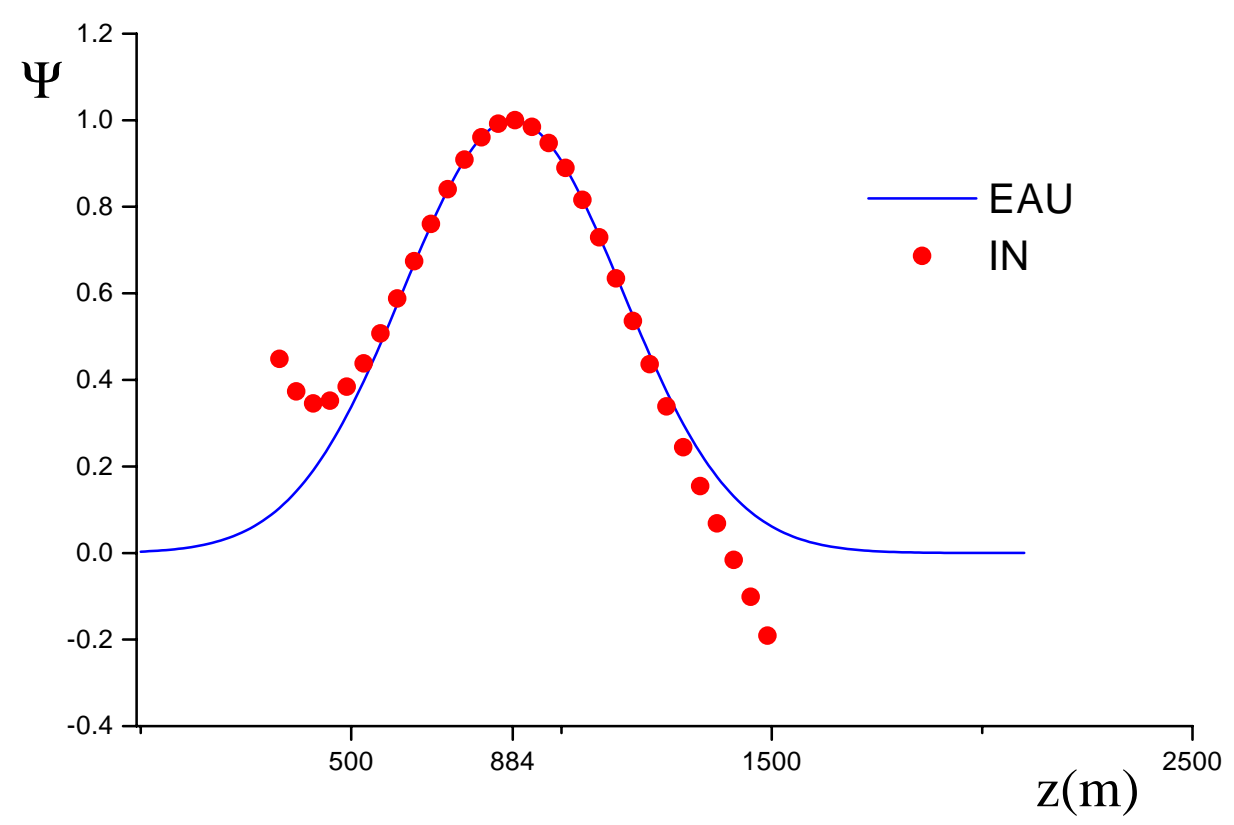

b)

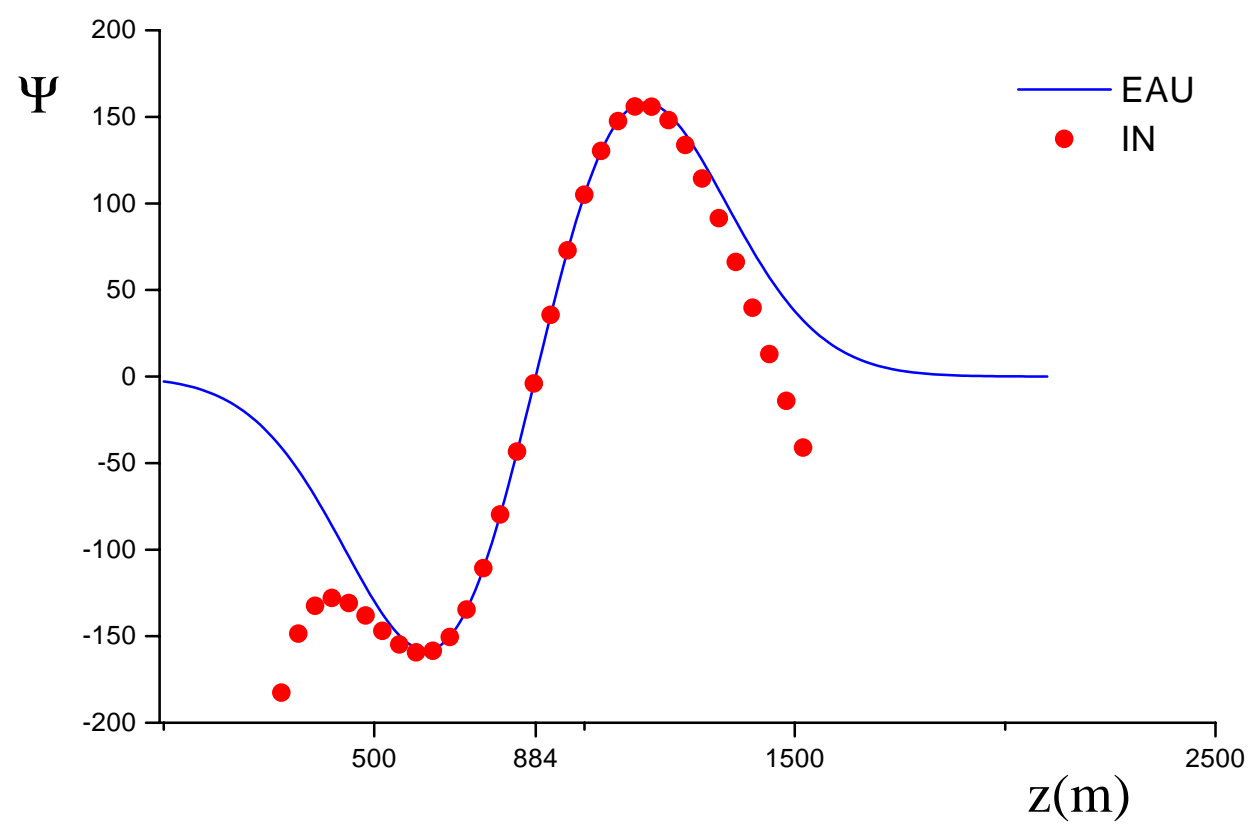

Figura 2: para uma freqüência $\omega=50 \mathrm{rad} / \mathrm{s}$, mostra em a) quando $n=0$ e em b) quando $n=1$, o comportamento do campo $\Psi$ (em unidades arbitrárias) em função da profundidade $z$. 